\title{
Mehrwertsteuerpflicht in Gruppenpraxen
}

\author{
R. Gmür, Rechtsdienst FMH
}

La version française suivra

\section{Einleitung und genereller Hinweis}

Die Mehrwertsteuer wurde in der Schweiz 1995 eingeführt. Da der Gesundheitsbereich, abgesehen von Arznei- und Hilfsmitteln, nur marginal davon betroffen ist, hat sich die Revisionstätigkeit der Eidgenössischen Steuerverwaltung bisher auf andere Branchen konzentriert. In jüngerer Zeit haben nun aber auch die ersten Kontrollen in Arztpraxen stattgefunden. Dabei ist es insbesondere in Gruppenpraxen zum einen oder anderen Problem oder sogar zu bösen Überraschungen gekommen: Es betrifft dies einerseits die Zuordnung von Medikamentenumsätzen, andererseits die reine Nutzung der Praxisinfrastruktur durch Ärztinnen und Ärzte, die nicht Mitinhaber der Gruppenpraxis sind.

Im nachfolgenden Beitrag werden die wesentlichen Fragen aufgeworfen und die Praxis der Steuerverwaltung dargestellt und kommentiert.

\section{Die wesentlichen Grundregeln}

\subsection{Steuerpflichtige Leistungen}

Ärztliche Dienstleistungen sind, wie das Gesundheitswesen generell, bekanntlich weitgehend von der Mehrwertsteuer ausgenommen. In der Arztpraxis praktisch relevante Ausnahme von der Ausnahme bildet die «Lieferung» (= Verkauf) von Medikamenten und Hilfsmitteln sowie in gewissen Konstellationen Entschädigungen für Infrastrukturleistungen.

\subsection{Die Steuerbefreiungsgrenzen}

Steuerpflichtig wird erst, wer mit steuerbaren Lieferungen oder Dienstleistungen eine bestimmte jährliche Umsatzgrenze überschreitet. Diese Steuerbefreiungsgrenze liegt bei einem Jahresumsatz von 75000 Franken, beziehungsweise 250000 Franken, wenn die Steuer nach Abzug der Vorsteuer weniger als 4000 Franken beträgt. Verschiedenartige Dienstleistungen und Lieferungen werden zusammengezählt, auch wenn sie verschiedenen Steuersätzen unterliegen. Für den Verkauf von Medikamenten kommt aufgrund des reduzierten Steuersatzes von $2,4 \%$ faktisch immer die zweite Steuerbefreiungsgrenze (250 000/4000) zur Anwendung; die ebenfalls steuerpflichtige Abgabe von Hilfsmitteln zum vollen Satz von 7,6\% fällt aufgrund

\section{Hinweis}

Die Ausführungen in diesem Beitrag sind gezwungenermassen relativ technisch und erschliessen sich auch dem aufmerksamen Leser nicht auf den ersten Blick. Falls überdies die Auseinandersetzung mit steuerrechtlichen Fragen nicht zu Ihren Lieblingsbeschäftigungen gehört, beachten Sie bitte zumindest folgenden Hinweis:

Wenn Sie im Rahmen einer Gruppenpraxis tätig sind und

- alle Gesellschafter zusammengezählt für mehr als Fr. 250000 Medikamente und Hilfsmittel umsetzen, individuell pro Gesellschafter aber unter dieser Grenze liegen;

- die Infrastruktur einem Praxispartner, der sich nicht in die Praxis eingekauft hat, gegen ein bestimmtes Entgelt zur Verfügung gestellt wird; oder

- für die gemeinsame Infrastruktur eine juristische Person («Innen-Gesellschaft», z.B. AG oder Genossenschaft) gegründet haben,

dann nehmen Sie umgehend mit Ihrem Treuhänder/Steuerberater Kontakt auf und klären Sie ab, ob die gesellschafts- und mehrwertsteuerrechtliche Situation optimal gestaltet ist.

der relativ geringen Menge in der Regel nicht ins Gewicht. Anders kann dies aussehen, wenn bedeutendere Infrastrukturleistungen an andere Ärzte, zum vollen Satz von 7,6\%, ins Spiel kommen (siehe unten Ziffer 5).

\subsection{Der Begriff der Gruppenpraxis}

Der Begriff «Gruppenpraxis» ist ebenso wie Gemeinschaftspraxis, Praxisgemeinschaft, Institut oder Zentrum etc. kein Rechtsbegriff, sondern umgangssprachlicher Natur. Für die gesellschaftsund steuerrechtliche Einordnung kommt es mit anderen Worten grundsätzlich nicht auf die zufällig gewählte Bezeichnung an, sondern auf die effektive rechtliche und organisatorische Ausgestaltung der Zusammenarbeit.

Die allermeisten Gruppenpraxen sind als mehr oder weniger weit gefasste Apparate- bzw. Infrastrukturgemeinschaften konzipiert. Recht- 
lich bilden diese Infrastrukturgemeinschaften, soweit sie nicht in Form einer juristischen Person (z. B. sogenannte Hintergrund-AG oder Hintergrund-Genossenschaft) konstituiert sind, eine einfache Gesellschaft. In neuer Zeit haben sich auch losere Zusammenarbeitsformen im Sinne der reinen Infrastrukturnutzung, ohne Einkauf in die Gruppenpraxis, entwickelt. Sodann gibt es den noch eher seltenen Fall der Gruppenpraxis in Form einer juristischen Person (v.a. AG), die im Gegensatz zur erwähnten Hintergrund- oder Infrastruktur-AG im Aussenverhältnis, gegenüber Patienten und Vertragspartnern, als solche auftritt. Und schliesslich gibt es nicht immer ganz übersichtliche Mischformen mit unterschiedlichem rechtlichem Status der beteiligten Ärzte.

\subsection{Die einfache Gesellschaft als Steuer- subjekt}

Anders als im Einkommenssteuerrecht bildet die einfache Gesellschaft im Mehrwertsteuerrecht nebst den einzelnen Gesellschaftern ein eigenes Steuersubjekt. Wenn nun also eine solche einfache Gesellschaft einerseits Medikamente bestellt, Apparate einkauft, Personal einstellt, und andererseits diese Infrastruktur den einzelnen Gesellschaftern zur Verfügung stellt bzw. Medikamente liefert, würden die entsprechenden Beiträge und Einzahlungen der einzelnen Gesellschafter nach der Logik des Steuersystems als Entgelt der Mehrwertsteuer unterliegen. Angesichts der generellen Steuerbefreiung für ärztliche Heilbehandlungen würde dies $\mathrm{zu}$ einer künstlichen Verteuerung der Dienstleistungen von Gruppenpraxen und somit einer offensichtlich unhaltbaren Benachteiligung führen. Der Gesetzgeber hat deshalb eine weitere Ausnahme gemacht für «Dienstleistungen von Gemeinschaften $[\ldots]$, soweit diese Dienstleistung anteilsmässig zu Selbstkosten an die Mitglieder für die unmittelbare Ausübung ihrer Tätigkeiten erbracht werden» (Art. 18, Ziffer 6 MWStG). Konkretisiert wird diese Bestimmung in Ziffer 11 der Branchenbroschüre Nr. 20 der Mehrwertsteuerverwaltung ${ }^{1}$.

\section{Infrastrukturgemeinschaften in Form der einfachen Gesellschaft: Massgebender Medikamentenumsatz}

1 Die Branchenbroschüre Nr. 20 «Gesundheitswesen» kann auf der Website der Mehrwertsteuerverwaltung heruntergeladen werden: http://www.estv. admin.ch/data/mwst/d/mwstg/ druckpdf/540-20-d.pdf.
Wie erwähnt ist der Verkauf von Medikamenten und Hilfsmitteln erst dann mehrwertsteuerpflichtig, wenn, unter Berücksichtigung der Pauschale von 5\% direkt angewandter Medikamente, eine jährliche Umsatzgrenze von Fr. 250000 überschritten wird. Wenn nun die
Gruppenpraxis Medikamente als einfache Gesellschaft einkauft und dem Patienten verrechnet, werden die Umsätze der einzelnen Gesellschafter zusammengefasst und die einfache Gesellschaft besteuert.

Dies spielt natürlich dann keine Rolle, wenn jeder einzelne Gesellschafter ohnehin für mehr als Fr. 250000 Medikamente pro Jahr umsetzt. Falls indes jeder oder ein einzelner Gesellschafter für sich gesehen unter dieser Grenze liegen würde und die Betroffenen den Kumulierungseffekt und damit die Mehrwertsteuer vermeiden möchten, muss der Medikamentenverkauf vollständig aus der Gesellschaft herausgelöst und ausschliesslich vom einzelnen Gesellschafter betrieben werden. $\mathrm{Ob}$ dies der Fall ist, beurteilt die Steuerverwaltung nach folgenden zwei Kriterien:

- Die Lieferrechnungen müssen persönlich auf den einzelnen Arzt ausgestellt sein.

- Die Medikamente müssen vom einzelnen Gesellschafter persönlich in Rechnung gestellt werden. Ob eine Dienstleistung, rechtlich gesehen, persönlich oder von der Gruppenpraxis erbracht wird, ist eine heikle Abgrenzungsfrage, auf die hier aber nicht weiter eingegangen wird. Praktisch stellt die Steuerverwaltung nämlich in erster Linie auf die Art der Verrechnung ab. Konkret: Die Honorare müssen auf ein persönliches Honorarkonto (und nicht ein gemeinsames Praxiskonto) eingezahlt werden. Auf der Honorarrechnung darf als Absenderadresse ausschliesslich der Name des Arztes stehen und keinerlei Hinweis auf die Gruppenpraxis, also keine Firmen- oder firmenähnliche Namen wie «Gemeinschaftspraxis Dr. Muster und Dr. Vorbild», «Praxis Elfenstrasse», Bezeichnungen wie «Zentrum für dieses» oder «Institut für jenes» usw. (Die Revisoren der Steuerverwaltung prüfen in erster Linie das Honorarrechnungsformular; auf der Praxistafel oder im Briefkopf der übrigen Korrespondenz kann ohne weiteres auf die Gruppenpraxis hingewiesen werden.)

\section{Kommentar}

Man kann die Praxis der Steuerverwaltung als spitzfindig bezeichnen, und die allfälligen administrativen Umstellungen mögen lästig sein. Die Vorgaben sind aber wenigstens klipp und klar und in der Regel mit vertretbarem Aufwand umsetzbar. Etwas höher ist dieser Aufwand für Gruppenpraxen, die im sogenannten «Poolsystem» organisiert sind, also alle Einnahmen auf ein gemeinsames Honorarkonto verbuchen; das ist vor allem bei Ehepartnergruppenpraxen der Fall. 
Sie müssten ihre Organisationsstruktur von Grund auf ändern, das heisst insbesondere eine getrennte Buchhaltung einführen (und einen in der Regel fehlenden schriftlichen Gesellschaftsvertrag abschliessen.) $\mathrm{Ob}$ sich das unter dem Strich lohnt, lässt man am besten professionell von seinem Treuhänder oder Steuerberater überprüfen. Nicht ganz aus den Augen verlieren sollte man dabei die Überlegung, dass die MWST fix in den SL-Preisen eingerechnet ist. Das Abführen der MWST ist mit anderen Worten der Normalfall; wer dank der Steuerbefreiungsgrenze davon verschont bleibt, generiert somit einen (bescheidenen) Zusatzertrag.

\section{Die Infrastrukturgemeinschaft in Form einer juristischen Person}

Ein Problem ergibt sich dann, wenn die Infrastrukturgemeinschaft nicht eine einfache Gesellschaft bildet, sondern in Form einer juristischen Person (z. B. AG oder Genossenschaft) ausgestaltet ist. Für sie gilt Ausnahme von Art. 18, Ziffer 6 MWSTG nicht, und die vertraglich festgelegten Kostenbeiträge der Aktionäre oder Genossenschafter an die Leistungen ihrer eigenen Gesellschaft sind vollumfänglich steuerpflichtig.

Diese sogenannten Innen-Gesellschaften waren und sind relativ selten. Es ist aber nicht ausgeschlossen, dass betroffenen Gesellschaftern die mehrwertsteuerrechtliche Situation gar nicht bewusst ist. Ihnen ist zur Vermeidung einer wirklich bösen Überraschung dringend zu empfehlen, ihre Lage professionell überprüfen zu lassen, die Innen-Gesellschaft unter Umständen umgehend aufzulösen und ihre Struktur in eine einfache Gesellschaft umzuwandeln.

\section{Kommentar}

Es liegt auf der Hand, dass die geltende Gesetzeslage unbefriedigend ist. $\mathrm{Ob}$ nun die einfache Gesellschaft Infrastrukturleistungen zu Selbstkosten weiterverrechnet oder ob dies eine von den Praxispartnern beherrschte juristische Person tut, ist von der Sache her letztlich ein und dasselbe. Es ist nicht einzusehen, wieso die Ausnahme, die der Gesetzgeber für die einfache Gesellschaft macht, nicht auch für juristische Personen gelten soll.

Die Gesetzeslage ist darüber hinaus auch wenig zukunftsträchtig. Gerade wieder im Zusammenhang mit der Aufhebung des Kontrahierungszwangs setzen Gesundheitspolitiker grosse Stücke auf die Entwicklung von sogenannten Ärztenetzwerken. Konsequenterweise sollte der Gesetzgeber dann aber auch die gesellschafts- und steuerrechtlichen Rahmenbedingungen schaffen, damit solche Strukturen effizient und ohne künstliche Verteuerung durch die MWST aufgebaut werden können. Einen ersten Schritt in diese Richtung geht die parlamentarische Initiative Widrig vom 6. März 2002 betreffend Leistungen von Managed-care-Organisationen. Sie betrifft aber lediglich die versicherungstechnischen Leistungen von Managed-care-Organisationen. Logischerweise müssen aber auch auf der Ebene der Infrastruktur für die Heilbehandlung die entsprechenden Steuerbefreiungen folgen, und zwar nicht nur für die Infrastrukturleistungen von (kasseneigenen) Managed-careOrganisationen, sondern generell von ärztlichen Gruppenpraxen und Ärztenetzwerken, welche mit den Kassen Zusammenarbeitsverträge abschliessen sollen. Konkret ist Art. 18 Ziffer 6 MWSTG so anzupassen, dass auch Dienstleistungen von Infrastrukturgemeinschaften in Form von juristischen Personen von der Mehrwertsteuer ausgenommen sind.

\section{Die Gruppenpraxis in Form der Infrastrukturbeteiligung}

In den letzten Jahren haben sich vermehrt Zusammenarbeitsformen entwickelt, in denen einer oder mehrere Partner sich nicht in die Infrastruktur einkaufen, sondern sie lediglich nutzen. ${ }^{2}$ Besonders beliebt und sinnvoll ist diese Form der Zusammenarbeit bei relativ kleinen Arbeitspensen des «Junior-Partners» oder etwa für eine Probezeit vor dem geplanten Einkauf in eine Gruppenpraxis. Normalerweise ist in dieser Konstellation der «Junior-Partner» in eigenem Namen und auf eigene Rechnung tätig und leistet im Innenverhältnis eine in der Regel pauschale Abgabe (Fixbetrag oder Umsatzabgabe) an den oder die Inhaber der Praxisinfrastruktur. Diese Abgaben bilden nach Auffassung der Steuerbehörde ein steuerpflichtiges Entgelt für das Zur-Verfügung-Stellen von Infrastruktur und Personal, und zwar zum vollen Satz von 7,6\%.

Immerhin entsteht die Mehrwertsteuerpflicht erst dann, wenn diese Abgaben die Steuerbefreiungsgrenzen überschreiten. In einer ärztlichen Gruppenpraxis sind deshalb faktisch zwei Fälle zu unterscheiden:

- In Praxen ohne Selbstdispensation fallen in aller Regel nur die Umsätze aus Hilfsmitteln und eben die geschilderten Infrastrukturabgaben an. Soweit die Summe dieser Beträge jährlich unter 75000 Franken liegt, bleibt der Praxisinhaber von der Mehrwertsteuer ausgenommen. 
- In Praxen mit Selbstdispensation ist der Praxisinhaber häufig schon aufgrund des Medikamentenumsatzes mehrwertsteuerpflichtig, womit dann unabhängig vom Betrag auch die Infrastrukturabgabe voll besteuert wird. Oder der Medikamentenumsatz des Praxisinhabers liegt zwar leicht unter der Steuerbefreiungsgrenze von 250000 Franken, überschreitet diese aber zusammen mit der Infrastrukturabgabe. In diesem Fall wird der Praxisinhaber dann für beide Umsätze steuerpflichtig.

In dieser Konstellation können folgende Massnahmen dazu beitragen, die Steuerpflicht zu reduzieren oder gar zu vermeiden: Zunächst einmal kann die Miete der Räumlichkeiten aus der Pauschalabgeltung ausgenommen werden und in einem separaten (Unter-)Mietvertrag geregelt werden. Das Vermieten von Immobilien nämlich ist - im Unterschied zu Mobilien - generell von der Mehrwertsteuer ausgenommen. Allerdings gilt das nur für Räume, die der Junior-Partner ausschliesslich selber nutzt, und nicht für gemeinsam genutzte Räume. Als zweites könnte, um den bedeutenderen Kostenposten anzuvisieren, eine auf die Anstellung des Personals beschränkte einfache Gesellschaft gegründet werden. Konkret müssen dafür ganz einfach die Arbeitsverträge mit dem Personal im Namen der einfachen Gesellschaft aller Partner abgeschlossen (und die Sozialversicherungsbeiträge im Namen aller Gesellschafter abgerechnet) werden, und die Aufteilung der Lohnkosten in einem auf diesen Zweck beschränkten und damit recht einfachen Gesellschaftsvertrag festgehalten werden. Als allenfalls steuerpflichtige Infrastrukturabgabe verbleibt dann nur noch die Miete für die zur Verfügung gestellten Apparate und Einrichtungsgegenstände.

Ein weiteres: Gruppenpraxen in der beschriebenen Ausformung werden von der Steuerverwaltung schon bei Vorliegen geringster Indizien (auch Bezeichnungen und Hinweisen auf Briefpapier und Praxistafel, in Inseraten und Telefonbuch usw.) als Einheit betrachtet. Die Ausscheidung des Medikamenten- und Hilfsmittelumsatzes von Junior-Partnern, wie oben in Ziffer 3 beschrieben, ist deshalb praktisch kaum zu bewerkstelligen, womit dann in jedem Fall der Gesamtumsatz der Gruppenpraxis für die Steuerpflicht massgebend ist.

\section{Kommentar}

Unbefriedigend ist auch hier, dass eine flexible und sinnvolle Form der Zusammenarbeit durch die mehrwertsteuerrechtlichen Rahmenbedingungen ernsthaft kompromittiert wird. Das kann in bestimmten Konstellationen so weit gehen, dass man ganz darauf verzichtet und andere Formen suchen muss, insbesondere die Anstellung als Praxisassistent (wobei dem wiederum in allen Kantonen ausser Zürich die zeitliche Beschränkung der berufsrechtlichen Bewilligung entgegensteht).

Richtigerweise sind Infrastrukturabgaben von Chef- und Belegärzten an das Spital von der Mehrwertsteuer ausgenommen (Ziffer 8.3.2 der Branchenbroschüre Nr. 20); alles andere würde angesichts des Kostendrucks im Gesundheitswesen zu einer ernsthaften Hypothek für das Belegund Chefarztsystem werden. Nicht nachvollziehbar ist hingegen, warum die gleichen Verhältnisse in freier Praxis von der Steuerverwaltung anders qualifiziert werden. Die Diskussion mit den Revisoren wird hier allerdings nicht viel nützen: Nach bisheriger Erfahrung mit der Eidgenössischen Steuerverwaltung muss man schon bereit sein, den Rechtsweg zu beschreiten. Zurzeit sind denn auch Rekursverfahren von Anwaltssozietäten hängig, deren Situation allerdings nur bedingt mit ärztlichen Gruppenpraxen vergleichbar ist. Trotzdem: Das letzte Wort dürfte hierzu noch nicht gesprochen sein.

\section{Die Gruppenpraxis in Form der juristischen Person}

Im Unterschied zu den in Ziffer 3 genannten «Innen-Gesellschaften» gibt es auch die (noch) seltenen Fälle von Gruppenpraxen und ärztlich geleiteten ambulanten Behandlungszentren, die als juristische Person konstituiert sind und im Aussenverhältnis, gegenüber Patienten und anderen Vertragspartnern, als solche auftreten. Solche Einrichtungen gelten gemäss der Praxis der Steuerverwaltung als «Zentren für ärztliche Heilbehandlung und Diagnostik» im Sinne von Art. 18 Ziffer 2 MWSTG, deren Dienstleistungen, abgesehen von Medikamenten und Hilfsmitteln, ebenfalls von der Mehrwertsteuer ausgenommen sind. Die detaillierten Voraussetzungen sind in Ziffer 5 der Branchenbroschüre Nr. 20 aufgezählt.

Als mehrwertsteuerlich massgebender Umsatz gilt in dieser Konstellation selbstverständlich immer der gesamte Medikamenten- und Hilfsmittelumsatz des Zentrums. 